\title{
The Effect of the Transfer Matrix Applied to MIMO Systems in the Analysis of Cardiovascular Dynamics
}

\section{Carlos Alvarez Picaza ${ }^{1 *}$, Julián I Veglia ${ }^{1}$, Ángel E Piacenza ${ }^{2}$, Albert D Valdez $^{1}$, Paola L Schlesinger ${ }^{1}$ and Juan A Chiozza ${ }^{1}$}

${ }^{1}$ Facultad de Ciencias Exactas, Universidad Nacional del Nordeste, Corrientes, Argentina

${ }^{2}$ Facultad de Medicina, Universidad Nacional del Nordeste, Corrientes, Argentina

*Corresponding Author: Carlos Alvarez Picaza, Facultad de Ciencias Exactas, Universidad Nacional del Nordeste, Corrientes, Argentina.
Received: May 15, 2021

Published: July 22, 2021

(C) All rights are reserved by Carlos Alvarez

Picaza., et al.

\begin{abstract}
MIMO systems allow us to work in the state-space without restrictions. The use of the Modern Control Theory let to specify new behaviors of complex structures. We describe the dynamics of the cardiovascular system by finding the input-output relationships in the state-space of a functional cardiac model, based on state equations and controllability criteria of control theory. The use of the transfer matrix applied to dynamic systems with many inputs and outputs allows addressing the treatment of cardiovascular dynamics with a different perspective. Based on the controllability criteria of dynamic systems we present an alternative method of analyzing the state of the arterial wall as a function of compliance. The unit step response of the multiple-input multiple-output system model illustrates the damping effect of the arterial wall to the pulsatility of the heart. In addition to verifying that hypertensive patients have less inertia of blood flow, it established that the internal controllability of the system can be affected as a function of input-output correlations.
\end{abstract}

Keywords: State-space; Transfer Function; Controllability

\section{Introduction}

A model is, by definition, an approximation of a system in terms of its representation [1]. Mathematical modeling is currently applied to physiology and medicine to support the activities of the scientist and the clinic workers. The hemodynamic characteristics of the cardiac system, including peripheral resistance, arterial compliance and the characteristic impedance of the proximal aorta, help to understand this system [2]. Strong interactions between characteristics of the vascular system make difficult a good understanding, although mathematical modeling and parameter estima- tion can help to understand system performance. A well-known model for operating on vascular features is the Windkessel model.

The Windkessel model defines the heart and the systemic arterial system as a closed hydraulic circuit comprising a water pump connected to a chamber. The water pumped into the chamber compresses the air inside and pushes the water out of the chamber, back to the pump. This allows us to simulate the elasticity and extensibility of the main artery, since the ventricle of the heart pumps blood towards it. This effect is known as arterial compliance, represented by capacitor $C$. The term compliance is the parameter that 
specifies the elastic nature of the blood vessels. It is defined as the incremental change in volume that would result from an incremental change in pressure.

The resistance that water encounters as it exits the Windkessel model and flows back to the pump, simulates the resistance to flow that blood encounters as it flows through the arterial tree from the major arteries, minor arteries, arterioles, and capillaries, due to the decrease in the diameter of the vessel.This resistance to flow $\mathrm{R}$ is known as peripheral resistance $[3,4]$.

We consider the four-element Windkessel model, first proposed by Burathi and Gnudi in 1982 [5]. This model is shown schematically in figure 1 and consists of a parallel connection of a resistor and capacitor. The resistance $\mathrm{R}$ represents the total peripheral resistance and the capacitor $C$ represents the compliance of the vessels. Another resistive element between the pump and the air chamber simulates the resistance to blood flow due to the aortic or pulmonary valve. $\mathrm{L}$ is an inertial element in parallel with the characteristic impedance. With this arrangement, the model can account for the inertia of the entire arterial system at low frequencies and, at mid and high frequencies, allows the characteristic impedance to come into play [6].

Other authors have modeled the cardiovascular system based on the Windkessel model of two, three or four elements, to estimate the parameters $[2,7]$, to simulate the pressure signal waveform [8] or, to study some specific characteristics [9,12].

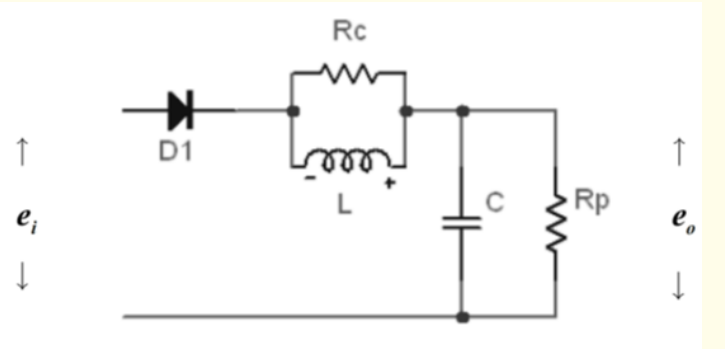

Figure 1: Four-element Winkessel model.

When considering multiple inputs and outputs, the dynamics of the system depends on the intervening transfer functions, there is no longer a single input-output relationship. The major arteries fulfill 2 different but interrelated functions: a) They constitute low resistance to blood distribution channels that deliver an adequate blood supply to the peripheral organs, called the conduction function and related to the static component of arterial pressure (mean arterial pressure), and b) Also, dampens pressure oscillations caused by the intermittent nature of ventricular ejection, called the damping function related to the pulsatile component (pulse pressure) [13]. Due to the damping function, the major arteries store a part of the stroke volume during systolic ejection (approximately $60 \%$ in normal conditions) and return it during diastole, losing $15 \%$ of the energy stored in heat (dissipated energy). This is known as the Windkessel effect, since it transforms the pulsatile flow of the central arteries into a continuous flow required by the peripheral tissues.

Our aim is to describe the dynamics of the cardiovascular system by searching for input-output relationships in the state space, assuming that the heart is a stable biological system with feedback $[14,15]$, determining its influence on the controllability of dynamic systems.

In this article we use space-state equations and controllability criteria to simultaneously calculate multiple relationships that characterize part of cardiac dynamics.

\section{Methods}

Data

The table 1 and 2 list the data corresponding to two categories of patients, with normal blood pressure and arterial hypertension (research group of Dr. Armentano - Favaloro University - Argentina). Columns 2 and 3 show the systolic and diastolic pressures. In column 4 , the average value of compliance. Column 5 indicates the pulse wave velocity. Column 6 lists the compliance calculated for each record. The values in column 7 denote the inertia of the system, a parameter that is calculated by subtracting the diastolic pressure from the systolic. In column 8 , the control pole, which will give the idea of stability of the dynamic system.

\section{Estimation of the conpliance}

Unlike what was published by Monzón., et al. [16], in this work we use the Branwell and Hill equation to find the compliance:

$$
C m=\frac{1334 . D m}{2 . r . P W V^{2}}
$$




\begin{tabular}{|l|c|c|c|c|c|c|c|}
\hline $\mathbf{N}^{\circ}$ & $\mathbf{S P}$ & $\mathbf{D P}$ & $\mathbf{A P}$ & $\mathbf{P W V}$ & $\mathbf{C m}$ & $\mathbf{L}$ & Pole \\
\hline & $\mathbf{m m H g}$ & $\mathbf{m m H g}$ & $\mathbf{m m H g}$ & $\mathbf{m} / \mathbf{s}$ & $\begin{array}{c}\mathbf{e - 4 c m} \mathbf{\mathbf { m }} / \\
\mathbf{m m g}\end{array}$ & $\mathbf{m m H g}$ & $\begin{array}{c}\text { (Con- } \\
\text { trol) }\end{array}$ \\
\hline 1 & 93 & 57 & 69 & 10,39 & 4,03 & 36 & $-1,1550$ \\
\hline 2 & 127 & 80 & 96 & 12,25 & 4,15 & 47 & $-1,1626$ \\
\hline 3 & 104 & 66 & 79 & 9,05 & 5,03 & 38 & $-1,2049$ \\
\hline 4 & 120 & 89 & 99 & 10,78 & 3,43 & 31 & $-1,1063$ \\
\hline 5 & 91 & 75 & 80 & 10,94 & 3,14 & 16 & $-1,0731$ \\
\hline 6 & 97 & 70 & 79 & 11,11 & 3,57 & 27 & $-1,1197$ \\
\hline 7 & 118 & 66 & 83 & 9,28 & 5,36 & 52 & $-1,2165$ \\
\hline 8 & 85 & 51 & 62 & 7,80 & 6,40 & 34 & $-1,2443$ \\
\hline 9 & 96 & 61 & 73 & 8,83 & 4,08 & 35 & $-1,1563$ \\
\hline 10 & 117 & 77 & 90 & 9,80 & 3,62 & 40 & $-1,1241$ \\
\hline 11 & 119 & 64 & 82 & 10,69 & 4,00 & 55 & $-1,1530$ \\
\hline
\end{tabular}

Table 1: Compliance and control pole for normotensive patients.

\begin{tabular}{|l|c|c|c|c|c|c|c|}
\hline $\mathbf{N}^{\circ}$ & $\mathbf{S P}$ & $\mathbf{D P}$ & $\mathbf{A P}$ & $\mathbf{P W V}$ & $\mathbf{C m}$ & $\mathbf{L}$ & Pole \\
\hline & $\mathbf{m m H g}$ & $\mathbf{m m H g}$ & $\mathbf{m m H g}$ & $\mathbf{m} / \mathbf{s}$ & $\begin{array}{c}\mathbf{e - 4} \mathbf{~ c m} \mathbf{~} \\
\mathbf{m m H g}\end{array}$ & $\mathbf{m m H g}$ & (Control) \\
\hline 1 & 146 & 96 & 113 & 15,21 & 2,00 & 50 & $\begin{array}{c}-0.68 \pm \\
0,77 \mathrm{i}\end{array}$ \\
\hline 2 & 106 & 84 & 91 & 14,58 & 2,21 & 22 & $-0,8139$ \\
\hline 3 & 116 & 65 & 82 & 10,07 & 3,49 & 51 & $-1,1122$ \\
\hline 4 & 157 & 89 & 112 & 11,15 & 3,76 & 68 & $-1,1357$ \\
\hline 5 & 166 & 98 & 121 & 14,25 & 2,68 & 68 & $-0,9948$ \\
\hline 6 & 164 & 92 & 116 & 16,26 & 2,22 & 72 & $-0,8216$ \\
\hline 7 & 127 & 82 & 97 & 11,44 & 3,76 & 45 & $-1,1357$ \\
\hline 8 & 155 & 92 & 113 & 17,16 & 2,48 & 63 & $-0,9417$ \\
\hline 9 & 155 & 70 & 98 & 10,83 & 3,70 & 85 & $-1,1309$ \\
\hline 10 & 139 & 100 & 113 & 11,28 & 4,04 & 39 & $-1,1557$ \\
\hline 11 & 134 & 84 & 101 & 10,27 & 4,82 & 50 & $-1,1965$ \\
\hline 12 & 114 & 75 & 88 & 11,31 & 3,12 & 39 & $-1,0704$ \\
\hline 13 & 117 & 78 & 91 & 14,07 & 2,31 & 39 & $-0,8753$ \\
\hline 14 & 125 & 83 & 97 & 14,78 & 1,68 & 42 & $-0,68 \pm$ \\
\hline & & & & & & & $0,35 i$ \\
\hline
\end{tabular}

Table 2: Compliance and control pole for hypertensive patients.

Where factors such as pulse wave velocity, blood viscosity, and artery diameter are involved.
The pulse wave velocity was calculated in a graphical way using the following formula:

$P W V=\frac{D}{\Delta x \cdot T}$

\section{Modeling in state-space}

As the operation of the cardiac system involves many variables such as pressure, blood density, compliance, resistance of the arterial wall, among others, it would be better to model it with a MIMO (Multiple Input - Multiple Output) system. We designed this model by connecting two SISO (Single Input - Single Output) systems in parallel. The input matrix represents two variables: complication and inertia of blood flow.

The transfer functions of the four-element Windkessel model with 2 inputs and 2 outputs are:

$$
\begin{aligned}
& s^{2} E_{o 1}(s)+s E_{o 1}(s) \frac{1}{R C}+E_{o 1}(s) \frac{1}{C}=E_{i 1}(s) \frac{1}{C} \\
& s^{2} L E_{o 2}(s)+s E_{o 2}(s) \tau+E_{o 2}(s)=E_{i 2}(s)
\end{aligned}
$$

Where $\tau$ is $\mathrm{RC}$.

From (1), the equations of the system model in the state space are defined by:

$$
\begin{aligned}
& \ddot{e}_{o 1}+\frac{1}{\tau} \dot{e}_{o 1}+\frac{1}{C} e_{o 1}=\frac{1}{C} e_{i 1} \\
& \ddot{e}_{o 2}+\frac{\tau}{L} \dot{e}_{o 2}+\frac{1}{L} e_{o 2}=\frac{1}{L} e_{i 2}
\end{aligned}
$$

The input, output and state variables of the system are:

$$
\begin{aligned}
& x_{1}=e_{o 1} ; \quad e_{i 1}=u_{1} \\
& x_{2}=\dot{e}_{o 1}=\dot{x}_{1} \\
& \dot{x}_{2}=-\frac{1}{C} x_{1}-\frac{1}{\tau} x_{2}+\frac{1}{C} u_{1} \\
& x_{3}=e_{o 2} ; e_{i 2}=u_{2} \\
& x_{4}=\dot{e}_{o 2}=\dot{x}_{3} \\
& \dot{x}_{4}=-\frac{1}{L} x_{3}-\frac{\tau}{L} x_{4}+\frac{1}{L} u_{2}
\end{aligned}
$$

$$
\begin{aligned}
& y_{1}=e_{o 1}=x_{1} \\
& y_{2}=e_{o 2}=x_{3}
\end{aligned}
$$


In matrix notation:

$$
\begin{aligned}
& \dot{\mathbf{x}}=\mathbf{A x}+\mathbf{B u} \\
& \mathbf{y}=\mathbf{C} \mathbf{x}
\end{aligned}
$$

Where $\dot{\mathbf{X}}$ is the state vector, $\mathbf{y}$ the output vector, A the state matrix, $\mathrm{B}$ the input matrix y $\mathrm{C}$ the output matrix.

The components of the state vector are represented by:

$$
\begin{aligned}
& \dot{x}_{1}=x_{2}=\dot{e}_{o 1} \\
& \dot{x}_{2}=-\frac{1}{C} x_{1}-\frac{1}{R C} x_{2}+\frac{1}{C} u_{1}+u_{2} \\
& \dot{x}_{3}=x_{4}=\dot{e}_{o 2} \\
& \dot{x}_{4}=-\frac{1}{L} x_{3}-\frac{\tau}{L} x_{4}+\frac{1}{L} u_{2}+u_{1}
\end{aligned}
$$

Finally,

$$
\begin{aligned}
& {\left[\begin{array}{l}
\dot{x}_{1} \\
\dot{x}_{2} \\
\dot{x}_{3} \\
\dot{x}_{4}
\end{array}\right]=\left[\begin{array}{cccc}
0 & 1 & 0 & 0 \\
-\frac{1}{C} & -\frac{1}{\tau} & 0 & 0 \\
0 & 0 & 0 & 1 \\
0 & 0 & -\frac{1}{L} & -\frac{\tau}{L}
\end{array}\right]\left[\begin{array}{l}
x_{1} \\
x_{2} \\
x_{3} \\
x_{4}
\end{array}\right]+\left[\begin{array}{cc}
0 & 0 \\
1 / C & 1 \\
0 & 0 \\
1 & 1 / L
\end{array}\right]\left[\begin{array}{l}
u_{1} \\
u_{2}
\end{array}\right]} \\
& {\left[\begin{array}{l}
y_{1} \\
y_{2}
\end{array}\right]=\left[\begin{array}{llll}
1 & 0 & 0 & 0 \\
0 & 0 & 1 & 0
\end{array}\right]\left[\begin{array}{l}
x_{1} \\
x_{2} \\
x_{3} \\
x_{4}
\end{array}\right]}
\end{aligned}
$$

\section{System controllability}

Systems analysis consists of two parts:

- Quantitative analysis, in which we are interested in the exact response of the system for a given set of inputs and initial conditions;

- Qualitative analysis, in which we are interested in the properties of the system.

Controllability is one of the qualitative properties of dynamic systems.

Broadly speaking, controllability studies the possibility of guiding the states of a system to a desired position by means of the input signal.

The pair $\{A, B\}$ are said to be controllable if for any initial state $x(0)=x_{0}$ and any final state $x_{1}$, there is an input $u$ that transfers $x_{0}$

to $\mathrm{x}_{1}$ in a finite time interval.

For the system to be controllable, is required that the dimension matrix $\mathrm{n}^{\prime} \mathrm{nr}$

$\mathscr{C}=\left[\begin{array}{llll}\mathbf{B} & \mathbf{A B} & \ldots & \mathbf{A}^{n-1} \mathbf{B}\end{array}\right]_{n \times n r}$

The rank of the matrix needs to be $=n$, that is, it contains $n$ linearly independent vectors; or that the determining factor is 10 . The matrix given in (10) is called the controllability matrix.

As a contribution to future work, by using the Controllability criteria, it is possible to develop other analyzes and find out new parameters, as well as the Pole Location Method and the Linear Quadratic Regulator, which indicate, in the first case, the vital work points for the patient (pacemaker regulation), and in the second, the operating points with less energy expenditure.

In addition, the stability of the system is closely related to the concept of Controllability. In the state space, Stability is a parameter that can be established and graphed.

\section{Results and Discussions}

The values found for the different control poles are listed in column 8 of table 1 and 2. These, the further away (left) from the ordinate axis in Laplace space (s), they will make the system more stable. According to table 2 - patient 14, which this hypertensive patient, having its conjugated poles, indicates that its damping factor is less than that of the normotensive patient, with respect to its frequency response.

$\mathrm{DC}_{N 8}=-0,24$
$\mathrm{D} \boldsymbol{C}_{H 16}=-3,54$

We found non-zero determinants of the 25 cases analyzed, therefore the "global" dynamic state of the patients is controllable.

Also all ranks of $\mathscr{C}=4$.

Also all ranks of the controllability matrix $=4$.

There is a unique relationship between compliance and the calculated pole. For high compliance values, the pole moves away from the ordinate axis in the Laplaces field. This indicates that for highly compliant systems, the model tends to be more stable.

We could calculate the relationships between all inputs and outputs due to the controllability of our system. As an example, we show the transfer matrices for the signals of patients 8 in table 1 and 14 in table 2, cases with the highest and lowest compliance 
calculated. Each element of the matrix is a transfer function that indicates the relationships between inputs and outputs for that particular patient.

The transfer function G (s) in Laplace space relates the output to the input. In the case of MIMO systems we have the relations $\mathrm{G}_{\text {output input }}(\mathrm{s})$.

For patient 8 of table 1:

$$
\begin{aligned}
& {\left[\begin{array}{l}
\mathbf{Y}_{N 1}(s) \\
\mathbf{Y}_{N 2}(s)
\end{array}\right]=\left[\begin{array}{ll}
\mathrm{G}_{N 11}(s) & \mathrm{G}_{N 12}(s) \\
\mathrm{G}_{N 21}(s) & \mathrm{G}_{N 22}(s)
\end{array}\right]\left[\begin{array}{l}
\mathbf{U}_{N 1}(s) \\
\mathbf{U}_{N 2}(s)
\end{array}\right]} \\
& \mathrm{G}_{N 11}=\frac{0,1563 s^{2}+0,0034 s+0,0046}{s^{4}+1,3913 s^{3}+0,2151 s^{2}+0,0436 s+0,0046} \\
& \mathrm{G}_{N 21}=\frac{s^{2}+1,3699 s+0,1563}{s^{4}+1,3913 s^{3}+0,2151 s^{2}+0,0436 s+0,0046} \\
& \mathrm{G}_{N 12}=\frac{s^{2}+0,0215 s+0,0294}{s^{4}+1,3913 s^{3}+0,2151 s^{2}+0,0436 s+0,0046} \\
& \mathrm{G}_{N 22}=\frac{0,0294 s^{2}+0,0403 s+0,0046}{s^{4}+1,3913 s^{3}+0,2151 s^{2}+0,0436 s+0,0046}
\end{aligned}
$$

For patient 14 of table 2 ,

$$
\begin{aligned}
& {\left[\begin{array}{r}
\mathbf{Y}_{H 1}(s) \\
\mathbf{Y}_{H 2}(s)
\end{array}\right]=\left[\begin{array}{ll}
\mathrm{G}_{H 11}(s) & \mathrm{G}_{H 12}(s) \\
\mathrm{G}_{H 21}(s) & \mathrm{G}_{H 22}(s)
\end{array}\right]\left[\begin{array}{l}
\mathbf{U}_{H 1}(s) \\
\mathbf{U}_{H 2}(s)
\end{array}\right]} \\
& \mathrm{G}_{H 11}=\frac{0,5952 s^{2}+0,0103 s+0,0142}{s^{4}+1,3872 s^{3}+0,6429 s^{2}+0,0430 s+0,0142} \\
& \mathrm{G}_{H 21}=\frac{s^{2}+1,3699 s+0,5952}{s^{4}+1,3872 s^{3}+0,6429 s^{2}+0,0430 s+0,0142} \\
& \mathrm{G}_{H 12}=\frac{s^{2}+0,0174 s+0,0238}{s^{4}+1,3872 s^{3}+0,6429 s^{2}+0,0430 s+0,0142} \\
& \mathrm{G}_{H 22}=\frac{0,0238 s^{2}+0,0326 s+0,0142}{s^{4}+1,3872 s^{3}+0,6429 s^{2}+0,0430 s+0,0142}
\end{aligned}
$$

Figure 2 illustrates how the input and output relationships are constructed.

The transfer functions, thus expressed in polynomial form do not give information about the controllability of the system.

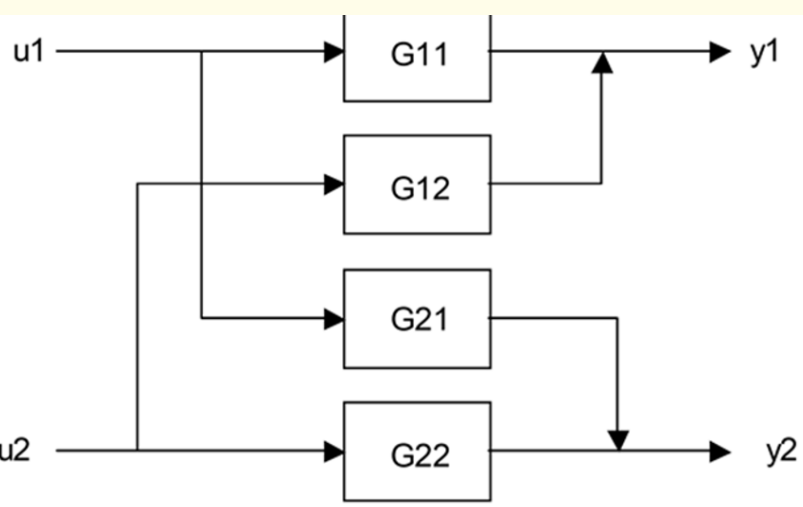

Figure 2: Multiple transfer functions.

The necessary and sufficient condition for the complete controllability of the state is that a cancellation does not occur in the transfer function or in the transfer matrix.

For this, we must express them in terms of their poles and zeros. If cancellation happens, the system cannot be controlled in the direction of canceled mode.

If we write it this way,

$$
\mathrm{G}(s)=\frac{K\left(s+z_{1}\right)\left(s+z_{2}\right) \ldots\left(s+z_{m}\right)}{\left(s+p_{1}\right)\left(s+p_{2}\right) \ldots\left(s+p_{n}\right)}, \quad \text { with } m<n
$$

We can find if there is any cancellation.

Of the eight transfer functions analyzed, it was found that the relationship $\frac{Y_{2}(s)}{U_{1}(s)}=\mathrm{G}_{21}(s)$ presents cancellations between poles and zeros, both for normotensive and hypertensive patients, this implies that the "internal" dynamic controllability of the system is compromised for these output/input relationships.

For the case of the normotensive system,

$$
\mathrm{G}_{N 21}=\frac{(s+1,244)(s+0,1256)}{(s+1,244)(s+0,1258)\left(s^{2}+0,02131 s+0,02939\right)}
$$

This indicates that for MIMO systems it is often not enough to calculate the determinant of the controllability matrix, since there are many interrelationships between inputs and outputs. The canceled transfer function does not carry all the information that characterizes the system [17]. 
Which is consistent with what is reflected in figure 3c. Cardiac wall relaxation times are low.

Figure 3 shows the response of the model for a normotensive and a hypertensive patient (Record 8 of table 1, record 14 of table 2 ). Figure 3a illustrates the variation of time compliance for systolic pressure as a function of its own output/input ratio, $\mathrm{Y}_{1}(\mathrm{~s}) /$ $\mathrm{U}_{1}(\mathrm{~s}) \rightarrow 1$, while $3 \mathrm{~b}$ ) represents the values found of the compliance - table 1 (6,40 e-4 cm/mmHg) and II (1,68 e-4 cm/mmHg) corresponding to $\mathrm{Y}_{1}(\mathrm{~s}) / \mathrm{U}_{2}(\mathrm{~s})$.

Figure $3 c$ reveals the variation of the inertial element for both categories of patient according to the considered recovery time. ( $\mathrm{RC}=730 \mathrm{msec}$.), being $34 \mathrm{mmHg}$ for the normotensive patient and $42 \mathrm{mmHg}$ for the hypertensive patient $\mathbb{B} \mathrm{Y}_{2}(\mathrm{~s}) / \mathrm{U}_{1}(\mathrm{~s})$ and figure $3 \mathrm{~d}$ show the inertia of blood flow over time for the systolic and diastolic pressures, respectively, $\mathrm{Y}_{2}(\mathrm{~s}) / \mathrm{U}_{2}(\mathrm{~s}) \rightarrow 1$ also in this case.

In figure $3 c$ the recovery time of the arterial wall, as shown by the inertia of blood flow, is approximately $30 \mathrm{msec}$, longer than the modeled recovery time for normotensive patients.

Compliance represents the increase in the volume of blood in a vessel when the pressure in the same vessel also increases. As is known, patients with arterial hypertension show reduced compliance and compliance compared to normotensive subjects, which supports our results $[18,19]$.

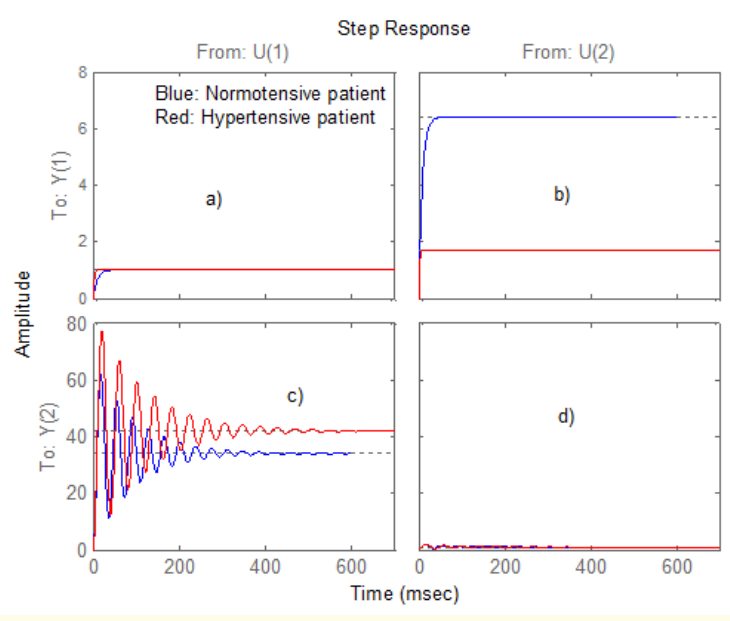

Figure 3: Response for the two categories of patients.
To enrich the results in the space of time, figure 4 represents the frequency response of the transfer functions $\mathrm{G}_{21}(s)$ from figure 3c), which, according to the calculations, shows an incomplete controllability of the system.

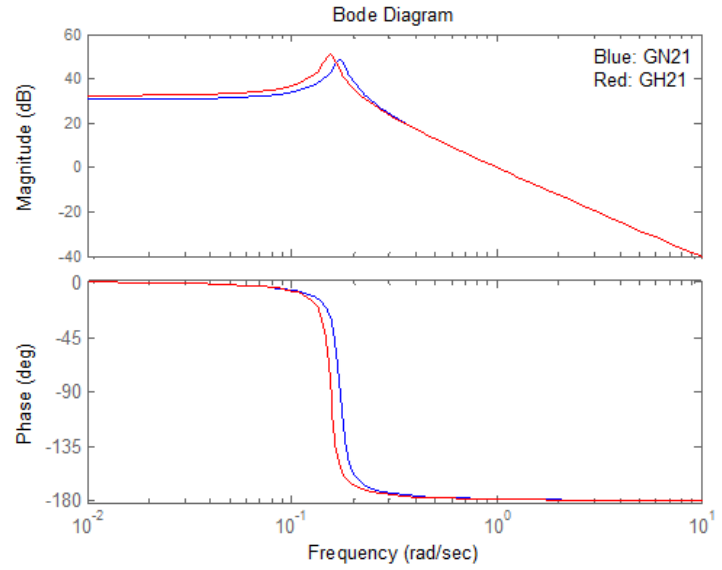

Figure 4: Frequency response of $G_{21}$.

It can be seen that the amplitude in frequency of the signal of the normotensive patient (blue) is less than that of the hypertensive (red). But the phase angle of the normotensive patient is greater than that of the hypertensive patient. This indicates that hypertensive patients have less inertia to blood flow.

To give an idea of the effect of compliance on the heart wall, we present the following graphs.

Compliance was considered as input values and the systolic and diastolic pressures as state variables.

In figure 5 is observed the dynamic behavior of the cardiac system of a normotensive patient. The damping of the arterial wall composed of the tandem $\mathrm{R}$ (peripheral resistance) $\mathrm{C}$ (compliance) is such that at the beginning of the next beat (approx. 1 second) the signals of both pressures (systolic and diastolic) do not overlap, which implicate normal operation.

Figure 6 shows how damping increases substantially, when looking at the times that are handled. Pressure signals tend to overlap before the next heartbeat starts (approx. 1 second). 


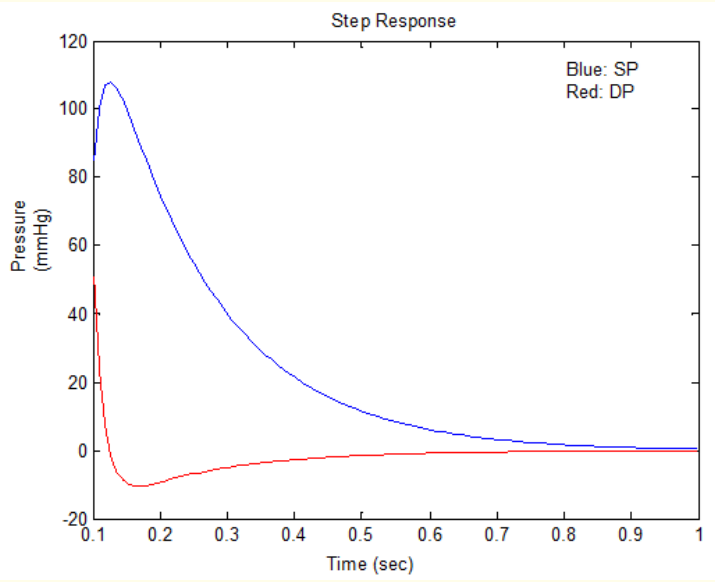

Figure 5: Dynamic Response - Arterial wall - Normotensive patient 8 - Table I.

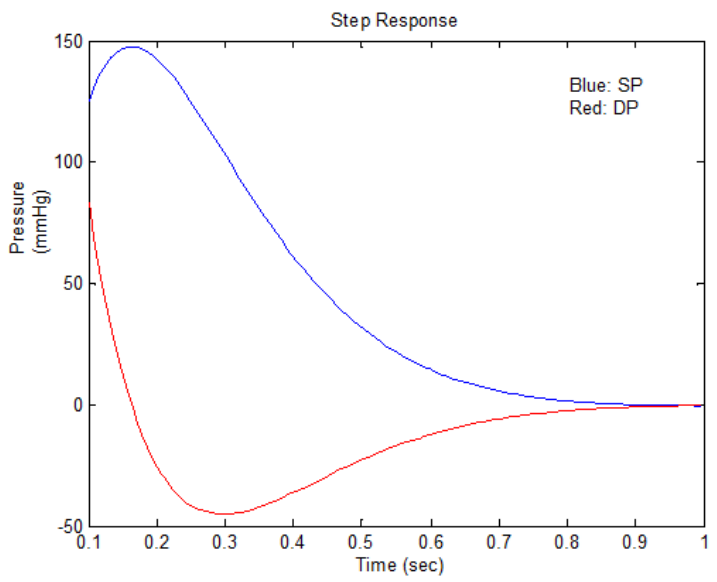

Figure 6: Dynamic Response - Arterial wall - Hypertensive patient 14 - Table 2.

\section{Conclusion}

Modeling the cardiac system with tools of the modern control theory, allows analysis of cardiovascular dynamics using equations of state and multiple transfer functions.

By using the controllability criteria, it was possible to establish that there may be critical correlations between some outputs and inputs, which was demonstrated by using this criterion applied to the transfer functions that define both systems.

The aim of our work was to find the relationship between arterial compliance and inertia of blood flow with systolic and diastolic pressures, two clinical variables that can be captured in an easy and non-invasive way into the hospital setting and that allowed the development of the analysis of cardiac systems by the conformation of the different transfer functions, which for a MIMO system would correspond to call them, the transfer matrix.

\section{Acknowledgment}

This work responds to the 18F011 Research Project funded by the General Secretariat of Science and Technology of the Universidad Nacional del Nordeste.

\section{Bibliography}

1. C Cobellia and E Carson. "Introduction to Modeling in Physiology". Academic Press, Elsevier. The Netherlands (2008).

2. T Kind., et al. "Estimation of Three- and Four-Element Windkessel Parameters Using Subspace Model Identification". IEEE Transactions on Biomedical Engineering 57.7 (2010): 15311538.

3. Y Nobuaki., et al. "Simulation Algorithm for the Coupling of the Left Ventricular Mechanical Model with Arbitrary Circulation Model". Proceedings of the 27th Annual International Conference of the IEEE-EMBS (2005): 7632-7635.

4. DR Kerner. "Solving Windkessel Models with MLAB".

5. R Burattini and G Gnudi. "Computer identification of models for the arterial tree input impedance: Comparison between two new simple models and first experimental results". Medical and Biological Engineering and Computing 20 (1982): 134144.

6. N Stergiopulos., et al. "The Four-Element Windkessel Model". Proceedings of the 18th Annual International Conference of the IEEE-EMBS (1996): 1715-1716.

7. A Capello and G Gnudi. "Analysis of the Arterial PressureVolume Curve in the Three-Element Windkessel Model". Proceedings of the Conference of Computers in Cardiology (1993): 385-388.

8. M Hlavác. "Windkessel model analysis in Matlab". 
9. C Zervides and DR Hose. "A simple Computational Model-Based Validation of Guyton's closed circuit analysis of the heart and the peripheral circulatory system". 27Th Annual International Conference of the IEEE-EMBS (2005): 5486-5489.

10. T A Parlikar., et al. "Model-Based Estimation of Cardiac Output and Total Peripheral Resistance". Computers in Cardiology 34 (2007): 379-382.

11. T A Parlikar and G C Verghese. "A Simple Cycle-Averaged Model for Cardiovascular Dynamics". Proceedings of the 27th of the Annual International Conference IEEE-EMBS (2005): 54905494.

12. L Zhong., et al. "A Non-Invasive System for Assessment of Aortic Stiffness in Clinical Practice". Proceedings of the 27th of the Annual International Conference IEEE-EMBS (2005): 22482251.

13. Grignola JC., et al. "Acute Pilmonary Hypertension: Protective Role of Vascular SmoothMuscle Activation". Revista Española de Cardiología 56.56 (2003): 1077-1084.

14. Monzón JE., et al. "Dynamic modeling of the vascular system in the state-space". Proceedings of the 32th Annual International Conference of the IEEE-EMBS (2010): 2612-2615.

15. JE Monzón., et al. "Blind source separation of electrocardiographic signals using system stability criteria". Proceedings of the 29th Annual International Conference of the IEEE-EMBS (2007): 3493-3496.

16. JE Monzón., et al. "A multiple-input multiple-output system for modeling the cardiac dynamics". Proceedings of the 33th Annual International Conference of the IEEE-EMBS (2011): 9781-4244-4122-8.

17. Ogata K. "Ingeniería de Control Moderna”. 5ta Edición Ed. Pearson (2010).

18. Z Liu., et al. "Estimation of the total arterial compliance: an improved method and evaluation of current methods". American Journal of Physiology - Heart 251.3 (1986): 588-600.
19. RS Reneman and APG Hoeks. "Arterial distensibility and compliance in hypertension". The Netherlands Journal of Medicine 47.4 (1995): 152-161.

\section{Volume 5 Issue 8 August 2021}

\section{(C) All rights are reserved by Carlos Alvarez Picaza., et al.}

